\title{
Comparison of an automated microfluidic immunoassay technology (BiolC, lab-on-chips) and ImmunoCAP assay. Lab-on-chips as a tool for specific IgE (slgE) detection
}

\author{
Izabela Szymczak-Pajor, Rafal Pawliczak \\ Department of Immunopathology, Faculty of Medicine, Medical University of Lodz, Lodz, Poland \\ Adv Dermatol Allergol 2020; XXXVII (1): 56-60 \\ DOI: https://doi.org/10.5114/ada.2020.93383
}

\begin{abstract}
Introduction: The measurement of clinically important specific $\lg E$ (slgE) antibody is pivotal for both diagnosis and management of allergy. Two methods may be distinguished depending on the number of antigens tested simultaneously: singleplex and multiplex. BiolC is a multiplex, advanced, automated microfluidic immunoassay system enabling simultaneous slgE measurement against multiple allergens. ImmunoCAP is a singleplex assay for slgE detection and gold standard method for diagnosis of allergy.

Aim: To compare and validate the diagnostic capability of a multiplex slgE assay - BiolC assay with a singleplex ImmunoCAP assay.

Material and methods: Using both BiolC assay and ImmunoCAP assay, the slgE level in serum samples from 20 allergic disease patients with respect to 33 allergens (16 inhalant allergens, 16 food allergens and 1 contact allergen) was measured. Receiver operating characteristic (ROC) area under the curve (AUC), qualitative and semiquantitative comparisons were performed to compare both slgE measurement methods using statistical analyses. Results: ROC AUC analysis showed similar sensitivity and specificity of BiolC assay and ImmunoCAP assay. In qualitative analysis, the negative and positive agreements were $100 \%$ equal for each allergen. Spearman's rank correlation coefficients identified very high positive correlations between two assays for all tested allergens $(p<0.001)$. Conclusions: The BiolC showed agreement with ImmunoCAP assay. Sensitivity and specificity of both assays are similar, thus they showed similar diagnostic performance. However, careful interpretation of obtained results is necessary in clinical applications because of methodological differences between these two systems.
\end{abstract}

Key words: allergens, allergen-specific IgE, microfluidic immunoassay.

\section{Introduction}

IgE-mediated allergic diseases such as drug and food allergies are characterized by an increased level of circulating allergen-specific (slgE) antibodies [1]. Thus, the detection of slgE is crucial for treatment, diagnosis and prevention of IgE-mediated allergic diseases. The appropriate detection of slgE, identification of the clinically relevant allergen and corresponding symptoms also play a key role in decisions regarding selection of allergen immunotherapy. Additionally, highly sophisticated judgement is required during allergen test selection and test results' interpretation because detection of slgE demonstrates only sensitization, not an allergy itself. The profiles of slgE sensitization may vary with respect to race, allergic dis- ease and cultural differences. Thus, culinary culture and biogeographic characteristics should be taken into consideration when designing test panels [2, 3]. Clinicians should also consider age of the patient, cross-reactivity of the allergen, exposure history with relevant manifestations in identifications of causative allergen(s) [4-6].

Laboratory and skin-based tests are available for slgE detection. In vitro detection is based on usage of singleplex or multiplex allergen slgE assays [7]. There are many differences between methods of IgE detection [5]. Thus, the understanding the characteristic of each tests including reliability, validity, strengths and limitations is important [8]. In vivo skin test is not a quantitative and difficult to be standardized method for detection of slgE.

Address for correspondence: Prof. Rafał Pawliczak, MD, PhD, Department of Immunopathology, Faculty of Medicine, Medical University of Lodz, 7/9 Zeligowskiego St, Building 2, Room 177, 90-752 Lodz, Poland, e-mail: rafal.pawliczak@csk.umed.lodz.pl Received: 27.03.2018, accepted: 24.08.2018. 
As opposed to skin tests, serological tests do not require patients to stop taking medications during testing. Moreover, examination of the patient might be performed immediately after anaphylaxis without the concern of allergic reactions. The serologic test is not related to recurrence of allergic reactions because of testing [8,9].

ImmunoCAP is a classical "gold-standard" singleplex slgE detection system provided by Thermo Fisher (ImmunoCAP, Uppsala, Sweden) characterized by reproducibility, reliability as well as good accordance with results of skin tests and allergen challenge in vivo. Due to improvement and development of various fluorescent enzyme immunoassays, ImmunoCAP is a reference method for measurement of slgE recommended by the World Health Organization [10, 11]. However, individual ImmunoCAP is capable of detection of slgE only against a single allergen, thus it is a quite expensive test for using in clinical settings [12]. BiolC is a multiplex, automated microfluidic-based immunoassay system for detecting and measuring of slgE in serum with the advantage of low sample volume [13].

\section{Aim}

The aim of the current study was to compare diagnostic capability of the automated microfluidic-based immunoassay system $\left(\right.$ BiolC $\left.^{\oplus}\right)$ with that of ImmunoCAP system - a gold standard method for diagnosis of allergy.

\section{Material and methods}

\section{Participants and serum samples}

Twenty participants (age: 1-34 years old; 11 female and 9 male) were enrolled in this study. The participants underwent routine slgE assessment. The study was carried out in January 2018. Serum was isolated from freshly drawn blood samples. Afterwards, isolated serum was aliquoted into two samples and then stored at $4^{\circ} \mathrm{C}$. In order to prevent bias occurrence between performed assay procedures, the ImmunoCAP and BiolC testing were carried out in a parallel fashion and blindly on the same day.

\section{slgE detection assay}

The ImmunoCAP (Thermo Fisher Scientific, Uppsala, Sweden) and BiolC assays were compared with respect to detection of slgE. Both tests were conducted according to manufacturers' instructions. Thirty-three allergens: 16 inhalant allergens (Dermatophagoides pteronyssinus, Dermatophagoides farinae, Alternaria alternata, vernal grass, cocksfoot, timothy, rye, alder, birch-tree, hazel, oak, mugwort, plantain, rape, cat, dog), 16 food allergens (egg white, cow's milk, cod, rice, peanut, soya, hazelnut, tomato, carrot, potato, egg yolk, $\alpha$-lactalbumin, $\beta$-lactoglobulin, casein, banana, mix of citrus) and 1 contact allergen (latex) were chosen for comparison. The cut-off for a positive result for both BiolC and ImmunoCAP assays was $0.35 \mathrm{kU} / \mathrm{l}$. The same class determination range was adopted for both assays and expressed as: 0.00-0.34 kU/l (class 0), 0.35-0.69 kU/l (class 1), 0.70$3.49 \mathrm{kU} / \mathrm{l}$ (class 2), 3.50-17.49 kU/l (class 3), 17.5-49.99 kU/l (class 4), 50.00-99.99 kU/l (class 5), and > $100 \mathrm{kU} / \mathrm{l}$ (class 6).

\section{Description of the device and procedure of the microfluidic-based immunoassay test}

Five reagent delivery channels are part of the microfluidic cartridge for automated assay for allergen screening. The role of these channels is to pump reagents to the common reaction zone from individual storage tanks. Allergen extracts are immobilized in the common reaction zone and finally go to the waste tank with all reaction by-products. During the assay procedure, $90 \mu$ of undiluted serum or plasma, $120 \mu$ l of premixed substrate, $450 \mu$ l of wash buffer, $120 \mu$ l of HRP conjugate (dilution: $1: 1000$ ) is added to the proper tanks within the cartridge using a micropipette. Then, the cartridge is inserted into the BiolC instrument and automated, chemiluminescent immunoassay is carried out in less than $30 \mathrm{~min}$. Solenoid actuators are a part of the instrument pumping reagents in the cartridge in order to carry out two-step ELISA within 10 min each. The temperature of reaction is controlled at $32^{\circ} \mathrm{C}$. When the final substrate is added, the chemiluminescence signals are imaged using a low-resolution CCD camera. Cartridges are discarded after use [14].

\section{Statistical analysis}

To compare the multiplex assay and singleplex assay, we used IBM SPSS Statistic version 23.0 (IBM Corp., Armonk, NY) for multiple statistical tests. First, ROC AUC analysis was carried out to compare the sensitivity and specificity of each method for each allergen. For qualitative analysis, total agreement ratios (agreement of detection results) were calculated [(number of both tests presenting a positive result + number of both tests presenting negative results)/total number of tests] [15]. Pearson's $\chi^{2}$ test was used for analysis of this agreement. Cohen's $\kappa$ analysis was also used for comparison of these categorical cross-table data. Kappa values were assessed and categorized as follows: 0.8-1.0 (almost perfect), 0.6-0.8 (substantial), 0.4-0.6 (moderate), 0.2-0.4 (fair) and < 0.2 (poor) [16]. Class assessment was compared by gamma analysis. A stronger association is observed when the gamma index is closer to 1 . For semiquantitative comparison between class determinations, Spearman's rank correlation analysis was used for classes 0-6 as well as Kruskal's gamma analysis. The coefficients of Spearman' correlation were classified as follows: 0.9-1.0 (very high positive correlation), 0.7-0.9 (high positive), 0.50.7 (moderate positive), 0.3-0.5 (low positive), < 0.3 (negligible) [17]. Intra-class correlation was also performed to assess the correlation between quantitatively expressed results of both tests. Good agreement between two methods was observed when values are closer to $1 . P<0.05$ showed statistical significance. 

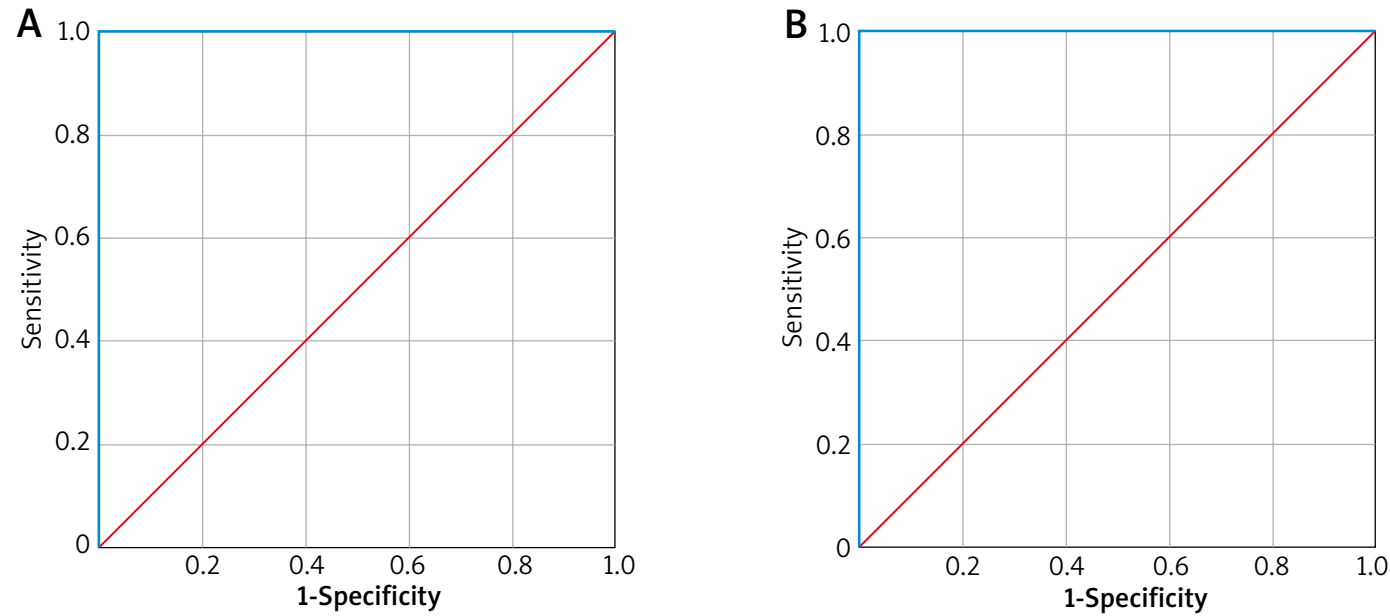

Figure 1. ROC curves for ImmunoCAP (A) and BiolC (B)

\section{Results}

\section{ROC analysis}

ROC AUC analysis was performed for each allergen and each method. ROC curves for the ImmunoCAP and BiolC are shown in Figure 1. Results of ROC AUC for all allergens for both assays are equal to 1 . Thus, both BiolC assay and ImmunoCAP assay as diagnostic methods have similar, very high sensitivity and specificity.

\section{Qualitative analysis: positivity and negativity}

The total diagnostic agreement ratios between assays - negativity and positivity for each allergen for each test are equal to 1 and are summarized in Table 1. Kappa index classifications were all 1 and determined as almost perfect. $P$-value calculated using Cohen's $\kappa$ analysis and Pearson's $\chi^{2}$ were all $<0.001$.

\section{Semi-quantitative analysis: class consistency (0-6 class)}

The analysis of class correlation data was performed using Spearman's correlations and gamma index. The gamma indexes and Spearman's correlation coefficients are equal to 1 . Gamma index values closer and equal to 1 indicate good agreement $(p<0.001)$. The class correlation analysis showed very high positive correlations between two assays for all tested allergens $(p<0.001)$.

\section{Quantitative comparison: intra-class correlation between two assays}

The analysis of intra-class correlations have indicated that values are equal to $1(p<0.05)$ for all tested allergens. Thus, correlation between quantitatively expressed results of both tests have shown good agreement.

\section{Discussion}

The main goal of the current study was to compare the diagnostic accuracy of the automated microfluidicbased immunoassay system (BiolC ${ }^{\oplus}$ known as lab-onchips and ImmunoCAP assay system - a laboratory method for identifying sensitization to specific allergens. Comparison of BiolC assay system and ImmunoCAP assay system was performed considering the difference of principles of the in vitro test and the in vivo test indicating a limitation of allergen skin test usage as a reference method [4]. ImmunoCAP assay is known for having established performance [18]. Thus, in our study we performed comparison with ImmunoCAP assay.

In the current study, BiolC assay showed almost perfect agreement in comparison with ImmunoCAP assay. Our results showed that two assays: singleplex ImmunoCAP assay and multiplex BiolC assay yield the same results using the same units but having differences in methodology. ROC AUC analysis showed that BiolC assay has similar sensitivity and specificity to ImmunoCAP assay.

High progress of nanotechnology has caused a wide application of microarrays both for routine outpatient settings and research. Currently, microarrays show great potential in the use for slgE level analysis. Moreover, microarrays are multitarget screening techniques (50+) with minimal serum (25-100 $\mu \mathrm{l})$ using for the test [14].

The microfluidic cartridge for allergen screening presented similar sensitivity to microarrays employing allergen extracts. Allergen extracts are a complex mixture of non-allergenic and allergenic proteins. Thus, the basis of slgE detection assay is high surface areas for immobilizing extracts constituting the challenge for miniaturizing assays. In accordance with our results, microfluidic cartridges present similar sensitivities as compared to other microassays that also employ allergen extracts [19-22]. Moreover, 
the advantage of microfluidics is capability to carry out reactions under dynamic (continuous flow or active mixing) rather than static conditions and provide more effective elimination of nonspecific bindings by non-allergenic proteins. From the point of view of medical centres, an automated operation and rapid centralized report system for test results obtained are needed. Protein-microarrays are multiple steps and prolonged manual operation takes 2-3 $\mathrm{h}$ to perform the test. In turn, BiolC assay is a multiplex, inexpensive assay with automated, rapid analysis intended for low-volume specific-allergen testing [14].

Some studies have compared singleplex and multiplex test results obtained from different assays. A study comparing the RIDA qLine Allergy (R-Biopharm AG, Darmstadt, Germany) with ImmunoCAP showed that it is more likely to provide false-positive results by RIDA system [23]. Comparison of three multiplex slgE assays (MAST Optigen allergy system (Hitachi Chemical Diagnostics), RIDA Allergy Screen, (R-Biopharm), Polycheck Allergy (Biocheck $\mathrm{GmbH}$ )) revealed that the Polycheck Allergy is more specific and MAST Optigen allergy system is more sensitive [24]. The comparison of AlloScreen with the skin prick test showed good agreement and correlation [25].

The insurance system and medical costs are different in each country. Thus, clinicians must be well-informed when taking into consideration weaknesses and strengths of available diagnostic methods considering their use in patient testing. The use of different assays for slgE detection is not guaranteed to obtain comparable results [26]. In vitro detection of slgE is not a guarantee of the allergic disease in a patient [5]. Numerous companies seek to improve the quality of in-vitro tests minimizing the disadvantages of their products [27]. Our results demonstrated that BiolC assay shows stronger agreement with ImmunoCAP. Shyur et al. also showed that microfluidic cartridge immunoassay has similar diagnostic accuracy to ImmunoCAP assay [13]. The presented BiolC system is a suitable diagnostic tool for decentralized testing where frequencies of the test are 16-20 per day. It is also possible that some improvements of these systems may be necessary. Currently, the technician is required to operate manual pipetting and reagent handling. The ideal solution would be storage of reagents on the cartridge itself provided that the period of cartridge self-life will be long [14].

The present study has some limitations. First, the number of participants enrolled in the study is low. Second, the test should be also carried out on non-allergic population. Notably, further studies on a larger number of participants and comparison with non-allergic population are needed.

Shyur et al. have presented a similar comparison as our study but made on a much larger number of patients. In the current study, we presented the comparison between results from BiolC assay and ImmunoCAP assay in the Polish population. The number of people suffering from allergy is still growing in Poland and finding new tools for fast slgE detection is needed.
Table 1. Positivity and negativity agreement between BiolC and ImmunoCAP

\begin{tabular}{ccccc}
\hline Allergens & $\begin{array}{c}\text { Agreement } \\
\text { with } \\
\text { ImmunoCAP }\end{array}$ & $\begin{array}{c}\text { Agreement } \\
\text { with }\end{array}$ & $\begin{array}{c}\text { Total } \\
\text { ImmunocAP }\end{array}$ & $\begin{array}{c}\text { Kappa } \\
\text { Imeement }\end{array}$ \\
& $(+)$ & $(-)$ & ratio
\end{tabular}

\begin{tabular}{lcccc}
\hline Dermatoph. pt. & $11 / 11(100 \%)$ & $9 / 9(100 \%)$ & 1 & 1 \\
\hline Dermatoph. far. & $10 / 10(100 \%)$ & $10 / 10(100 \%)$ & 1 & 1 \\
\hline $\begin{array}{l}\text { Alternaria } \\
\text { alternata }\end{array}$ & $4 / 4(100 \%)$ & $16 / 16(100 \%)$ & 1 & 1
\end{tabular}

\begin{tabular}{lllll}
\hline Vernal grass & $13 / 13(100 \%)$ & $7 / 7(100 \%)$ & 1 & 1 \\
\hline Cocksfoot & $13 / 13(100 \%)$ & $7 / 7(100 \%)$ & 1 & 1
\end{tabular}

\begin{tabular}{lllll}
\hline Timothy-grass & $13 / 13(100 \%)$ & $7 / 7(100 \%)$ & 1 & 1 \\
\hline
\end{tabular}

\begin{tabular}{lllll}
\hline Rye & $12 / 12(100 \%)$ & $8 / 8(100 \%)$ & 1 & 1 \\
\hline
\end{tabular}

\begin{tabular}{lllll}
\hline Alder & $6 / 6(100 \%)$ & $14 / 14(100 \%)$ & 1 & 1 \\
\hline Birch-tree & $8 / 8(100 \%)$ & $12 / 12(100 \%)$ & 1 & 1
\end{tabular}

\begin{tabular}{lllll} 
Birch-tree & $8 / 8(100 \%)$ & $12 / 12(100 \%)$ & 1 & 1 \\
\hline Hazel & $3 / 3(100 \%)$ & $17 / 17(100 \%)$ & 1 & 1 \\
\hline Oak & $2 / 2(100 \%)$ & $18 / 18(100 \%)$ & 1 & 1
\end{tabular}

\begin{tabular}{lllll}
\hline Mugwort & $3 / 3(100 \%)$ & $17 / 17(100 \%)$ & 1 & 1 \\
\hline Plantain & $3 / 3(100 \%)$ & $17 / 17(100 \%)$ & 1 & 1 \\
\hline
\end{tabular}

\begin{tabular}{lllll}
\hline Rape & $3 / 3(100 \%)$ & $17 / 17(100 \%)$ & 1 & 1 \\
\hline
\end{tabular}

\begin{tabular}{lcccc}
\hline Cat & $11 / 11(100 \%)$ & $9 / 9(100 \%)$ & 1 & 1 \\
\hline Dog & $10 / 10(100 \%)$ & $10 / 10(100 \%)$ & 1 & 1 \\
\hline
\end{tabular}

\begin{tabular}{lcccc}
\hline Dog & $10 / 10(100 \%)$ & $10 / 10(100 \%)$ & 1 & 1 \\
\hline Latex & $2 / 2(100 \%)$ & $18 / 18(100 \%)$ & 1 & 1
\end{tabular}

\begin{tabular}{lllll}
\hline Egg white & $2 / 2(100 \%)$ & $18 / 18(100 \%)$ & 1 & 1 \\
\hline
\end{tabular}

\begin{tabular}{lllll}
\hline Cow milk & $5 / 5(100 \%)$ & $15 / 15(100 \%)$ & 1 & 1 \\
\hline Cod & $1 / 1(100 \%)$ & $19 / 19(100 \%)$ & 1 & 1
\end{tabular}

\begin{tabular}{lllll} 
Cod & $1 / 1(100 \%)$ & $19 / 19(100 \%)$ & 1 & 1 \\
\hline Rice & $1 / 1(100 \%)$ & $19 / 19(100 \%)$ & 1 & 1 \\
\hline Peanut & $3 / 3(100 \%)$ & $17 / 17(100 \%)$ & 1 & 1 \\
\hline Soya & $1 / 1(100 \%)$ & $19 / 19(100 \%)$ & 1 & 1 \\
\hline Hazelnut & $2 / 2(100 \%)$ & $18 / 18(100 \%)$ & 1 & 1 \\
\hline Tomato & $1 / 1(100 \%)$ & $19 / 19(100 \%)$ & 1 & 1 \\
\hline Carrot & $1 / 1(100 \%)$ & $19 / 19(100 \%)$ & 1 & 1 \\
\hline Potato & $2 / 2(100 \%)$ & $18 / 18(100 \%)$ & 1 & 1 \\
\hline Egg yolk & $1 / 1(100 \%)$ & $19 / 19(100 \%)$ & 1 & 1 \\
\hline$\alpha$-Lactoalbumin & $1 / 1(100 \%)$ & $19 / 19(100 \%)$ & 1 & 1 \\
\hline$\beta$-Lactoglobulin & $1 / 1(100 \%)$ & $19 / 19(100 \%)$ & 1 & 1 \\
\hline Casein & $1 / 1(100 \%)$ & $19 / 19(100 \%)$ & 1 & 1 \\
\hline Banana & $1 / 1(100 \%)$ & $19 / 19(100 \%)$ & 1 & 1 \\
\hline Citrus mix & $1 / 1(100 \%)$ & $19 / 19(100 \%)$ & 1 & 1 \\
\hline
\end{tabular}

All p-values were $<0.001$.

\section{Conclusions}

The microfluidic cartridge immunoassay for detection of slgE is a promising instrument for both research and routine outpatient settings. BiolC assay has numerous advantages such as easy access, automated system 
with an extended working range and diagnostic sensitivity similar to ImmunoCAP assay. Additionally, BiolC system showed potential for decentralization of allergen screening as well as near-patient screening where rapid analysis, low instrumentation cost and ease of use are crucial in the clinical laboratory.

\section{Conflict of interest}

The authors declare no conflict of interest.

\section{References}

1. Johansson SGO, Bieber T, Dahl R, et al. Revised nomenclature for allergy for global use: Report of the Nomenclature Review Committee of the World Allergy Organization, October 2003. J Allergy Clin Immunol 2004; 113: 832-6.

2. Burney PGJ, Potts J, Kummeling I, et al. The prevalence and distribution of food sensitization in European adults. Allergy 2014; 69: 365-71.

3. Bousquet PJ, Chinn S, Janson C, et al. Geographical variation in the prevalence of positive skin tests to environmental aeroallergens in the European Community Respiratory Health Survey I. Allergy 2007; 62: 301-9.

4. Cox L, Williams B, Sicherer S, et al. Pearls and pitfalls of allergy diagnostic testing: report from the American College of Allergy, Asthma and Immunology/American Academy of Allergy, Asthma and Immunology Specific IgE Test Task Force. Ann Allergy Asthma Immunol Off Publ Am Coll Allergy Asthma Immunol 2008; 101: 580-92.

5. Park KH, Lee J, Lee SC, et al. Comparison of the ImmunoCAP Assay and AdvanSure ${ }^{T M}$ AlloScreen Advanced Multiplex Specific IgE Detection Assay. Yonsei Med I 2017; 58: 786-92.

6. Park KH, Lee J, Sim DW, Lee SC. Comparison of singleplex specific IgE detection immunoassays: ImmunoCAP Phadia 250 and Immulite 2000 3gAllergy. Ann Lab Med 2018; 38: 23-31.

7. Lee JH, Park HJ, Park KH, et al. Performance of the PROTIATM Allergy- $\mathrm{Q}^{\oplus}$ System in the detection of allergen-specific IgE: a comparison with the ImmunoCAP ${ }^{\oplus}$ System. Allergy Asthma Immunol Res 2015; 7: 565-72.

8. Bernstein IL, Li JT, Bernstein DI, et al. Allergy diagnostic testing: an updated practice parameter. Ann Allergy Asthma Immunol 2008; 100 (3 Suppl 3): S1-148.

9. Bousquet J, Heinzerling L, Bachert C, et al. Practical guide to skin prick tests in allergy to aeroallergens. Allergy 2012; 67: 18-24.

10. Crameri R. The crux with a reliable in vitro and in vivo diagnosis of allergy. Allergy 2013; 68: 693-4.

11. Lee JH, Park KH, Kim HS, et al. Specific IgE measurement using AdvanSure system: comparison of detection performance with ImmunoCAP ${ }^{\oplus}$ system in Korean allergy patients. Clin Chim Acta 2012; 413: 914-9.

12. Ollert M, Weissenbacher S, Rakoski J, Ring J. Allergen-specific IgE measured by a continuous random-access immunoanalyzer: interassay comparison and agreement with skin testing. Clin Chem 2005; 51: 1241-9.

13. Shyur SD, Jan RL, Webster JR, et al. Determination of multiple allergen-specific IgE by microfluidic immunoassay cartridge in clinical settings. Pediatr Allergy Immunol 2010; 21: 623-33.

14. Tai LW, Tseng KY, Wang ST, et al. An automated microfluidic-based immunoassay cartridge for allergen screening and other multiplexed assays. Anal Biochem 2009; 391: 98-105.
15. Lee YW, Sohn JH, Lee JH, et al. Allergen-specific IgE measurement with the IMMULITE 2000 system: intermethod comparison of detection performance for allergen-specific IgE antibodies from Korean allergic patients. Clin Chim Acta 2009; 401: 25-32.

16. Landis JR, Koch GG. The measurement of observer agreement for categorical data. Biometrics 1977; 33: 159-74.

17. Mukaka MM. Statistics corner: a guide to appropriate use of correlation coefficient in medical research. Malawi Med J J Med Assoc Malawi 2012; 24: 69-71.

18. Williams P, Sewell WC, Bunn C, et al. Clinical immunology review series: an approach to the use of the immunology laboratory in the diagnosis of clinical allergy. Clin Exp Immunol 2008; 153: 10-8.

19. Jahn-Schmid B, Harwanegg C, Hiller R, et al. Allergen microarray: comparison of microarray using recombinant allergens with conventional diagnostic methods to detect allergen-specific serum immunoglobulin E. Clin Exp Allergy J Br Soc Allergy Clin Immunol 2003; 33: 1443-9.

20. Bacarese-Hamilton T, Mezzasoma L, Ingham C, et al. Detection of allergen-specific IgE on microarrays by use of signal amplification techniques. Clin Chem 2002; 48: 1367-70.

21. Fall BI, Eberlein-König B, Behrendt $\mathrm{H}$, et al. Microarrays for the screening of allergen-specific IgE in human serum. Anal Chem 2003; 75: 556-62.

22. Kim TE, Park SW, Cho NY, et al. Quantitative measurement of serum allergen-specific lgE on protein chip. Exp Mol Med 2002; 34: 152-8.

23. Hwang H, Kwon J, Kim JY, et al. The RIDA Allergy Screen Versus the Phadiatop Test in 430 consecutive patient specimens. Lab Med 2016; 47: 20-9.

24. Han M, Shin S, Park H, et al. Comparison of three multiple allergen simultaneous tests: RIDA allergy screen, MAST optigen, and polycheck allergy. BioMed Res Int 2013; 2013 : 340513.

25. Cho JH, Suh JD, Kim JK, et al. Correlation between skin-prick testing, individual specific lgE tests, and a multiallergen IgE assay for allergy detection in patients with chronic rhinitis. Am J Rhinol Allergy 2014; 28: 388-91.

26. Szeinbach SL, Barnes JH, Sullivan TJ, Williams PB. Precision and accuracy of commercial laboratories' ability to classify positive and/or negative allergen-specific IgE results. Ann Allergy Asthma Immunol 2001; 86: 373-81.

27. Kim JK, Yoon YM, Jang WJ, et al. Comparison study between MAST CLA and OPTIGEN. Am J Rhinol Allergy 2011; 25: e156-9. 